\title{
Allometric relations between biomass and diameter at breast height and height of tree in natural forests at Me Linh Station for Biodiversity, Vinh Phuc Province, Vietnam
}

\author{
Mối tương quan giũa sinh khối với hai nhân tố đường kính và chiều cao cây \\ của cây gố trong các quần xã thực vật tự nhiên tại Trạm Đa dạng Sinh học Mê \\ Linh, tỉnh Vĩnh Phúc, Việt Nam \\ Research article
}

Dang Thi Thu Huong*, Do Huu Thu, Trinh Minh Quang, Nguyen Hung Manh, Bui Thi Tuyet Xuan, Nguyen Tien Dung

Institute of Ecology and Biological Resources, Vietnam Academy of Science and Technology, 18 Hoang Quoc Viet, Cau Giay, Ha Noi, Vietnam

\begin{abstract}
Stem diameter at breast height $\left(\mathrm{D}_{1.3 \mathrm{~m}}\right)$ and tree height $(\mathrm{H})$ are commonly used measures of tree growth. Based on correlation analysis between biomass of stem, branches and leaves and stem diameter and height of tree we can identify allometric equation for predicting biomass and carbon sequestration of the vegetation. This study was carried out in the natural forests of Me Linh Station for biodiversity to develop allometric equation between biomass and diameter at breast height and height of tree. The study results indicated that twenty tree species dominate in natural forests in Me Linh Station for Biodiversity and they were selected for sampling. Through the 80 established linear equation models for above and below -ground biomass (AGB and BGB), we found that the biomass of tree species in Me Linh Station for Biodiversity were closely correlated with the diameter factor $(\mathrm{R}>0.902)$ and not clearly correlated with the height (correlation coefficient $=0.5498$, $\left.\mathrm{R}^{2}<0.549\right)$. Four regression equations were established, including: $\mathrm{P}_{\text {stem }}=25.3051^{*}\left(\mathrm{D}_{1.3 \mathrm{~m}}\right)^{0.4627}$ $\left(R^{2}\right.$ : 9.661); $P_{\text {branch }}=12.1043 *\left(D_{1.3 m}\right)^{0.5416}\left(R^{2}: 9.8\right) ; P_{\text {leaves }}=9.446 *\left(D_{1.3 m}\right)^{0.5976}\left(R^{2}: 0.9363\right) ; P$ total biomass of forest $=25.882 * \mathrm{D}^{1.725}$ with $\left.\mathrm{R}^{2}: 0.8561\right)$ for estimating biomass and carbon sequestration of natural forest at the research site.
\end{abstract}

Đường kính ngang ngực $\left(D_{1.3 m}\right)$ và chiều cao $(H)$ cây là hai nhân tố thường được dùng để đánh giá sự phát triến của cây gố. Việc xây dưng các phuoong trình tuơng quan giữa sinh khối (SK) thân, cành, lá, sinh khối tầng cây gỗ, sinh khối của quần xã thực vật với đuoòng kinh và chiều cao cây góp phần rất lớn trong dụ báo sinh khối và khả năng hấp thụ khí carbon của thảm thục vật. Kết quả nghiên cứu cho thấy 20 loài cây gố chiếm uu thế trong rùng tư nhiên và chúng được chọn để thu mẫu. Mối tuơng quan giũa sinh khối với 2 nhân tố điều tra rùng là đường kính ngang ngực và chiều cao cây đã đươc kiểm tra thông qua 80 phuoong trình tuoong quan. Nhì chung, sinh khối có tuơng quan chặt chẽ với nhân tố đuờng kính (hệ số tuơng quan $R>0,902)$, và không tuoong quan rố với nhân tố chiều cao $(R<0,5498)$. Bốn phuơng trình tính sinh khối cho thảm rừng tại khu vưc nghiên cứu đã được thiết lạp: $S K_{\text {thân }}=25,3051 *\left(D_{1,3 m}\right)^{0,4627}\left(R^{2}: 9,661\right) ; S K_{\text {cành }}$ : $12,1043^{*}\left(D_{1,3 m}\right)^{0,5416}\left(R^{2}: 9,8\right) ; S K_{l a ̈}: 9,446^{*}\left(D_{l, 3 m}\right)^{0,5976}\left(R^{2}: 0,9363\right)$ và SKtồng $=25,882 * D^{1,725}$ with $\left.R^{2}: 0,8561\right)$.

Keywords: height, diameter, allometric, tree biomass equation, correlation coefficient $\left(\mathrm{R}^{2}\right)$ 


\section{Introduction}

Biomass is the most important indicator which aims to assess the productivity of a forest stand, it is also used to calculate the capacity and dynamics of forest carbon sequestration. Therefore, Brown \& Lugo (1992)[3] pointed out that, the biomass survey is also a part of the carbon sequestration study. The current focus of scientists is to develop an appropriate method to assess the value of forest in use and for predicting the ability of biomass accumulation as well as $\mathrm{CO}_{2}$ absorption in a forest ecosystem. From that point, a sustainable forest management and utilization could be established. At the present, methods of surveying and collecting samples for the calculation of forest biomass can be done, but they are difficult task and labor intensive, especially for the identification of natural forest production (Raae K., 2010; Timothy R.H., 2007) $[9,10]$.

Diameter at breast height and height of tree are measurements that can be obtained without high labour investment. Stem diameter is used either as a single inputvariable or in combination with height or wood density. The studies suggested that the use of regional heightdiameter models introduces significant biases in above ground biomass estimates, and that different heightdiameter models might be preferred for different forest types and they will be applied in future studies. However, there is a debate on which type of model should be selected to build a local site-specific height-diameter model. Biomass of the tree was estimated by extracting core samples at the breast height and mid height of the tree. When tested, there was no significant difference of density along the stem. Therefore, stem biomass was estimated using the density at breast height. Besides, there was no significant density difference between three sites. The study of allometry is extremely important in dealing with measurements and data analysis in the practice of forestry. Allometry studies the relative size of organs or parts of organisms. Tree allometry narrows the definition to applications involving measurements of the growth or size of trees. Allometric relationships are often used to estimate difficult tree measurements, such as volume, from an easily measured attribute such as $D_{1.3 m}$ (Bảo Huy, 2012) [2].

The regression analysis was used with pooled data to find out the relationship between the stem biomass with the tree diameter and height. Using variables of total height $(\mathrm{H})$, diameter at breast height $\left(\mathrm{D}_{1.3 \mathrm{~m}}\right)$ and their transformations, several models were established. The best model was selected by testing for the $\mathrm{R}^{2}$ value, standard residual distribution, modelling efficiency and bias (IPPC, 2006; Brown, 1992; FAO, 2004) [3,5,7).

Studying and exploring the correlation equations between biomass and surveying factors $\left(\mathrm{D}_{1.3 \mathrm{~m}}\right.$ and $\left.\mathrm{H}\right)$ to collect the actual a production basis, hence it would be used for inventorying and predicting forest biomass of typical vegetation in the Me Linh Biodiversity Stations, Vinh Phuc province.

\section{Materials and methods}

\subsection{Study sites}

The study is conducted in secondary forests of Me Linh Biodiversity Station, Vinh Phuc province, Vietnam, which is located in $21^{0} 23^{\prime} 57^{\prime}$ ' $-21^{0} 25^{\prime} 35^{\prime}$ 'S latitude and $105^{\circ} 42^{\prime} 40^{\prime}$ ' - $105^{\circ} 46^{\prime} 65^{\prime}$ ' E longitude (Fig. 1), distributed between $100 \mathrm{~m}$ and $500 \mathrm{~m}$ above sea level (a.s.1) with total area of about $178 \mathrm{ha}$ and is adjacent to Tam Dao National Park. According to report of Vinh Phuc province in 2010, the average annual precipitation is about $1358.7 \mathrm{~mm}$, temperature is $23.9^{\circ} \mathrm{C}$ and it has tropical monsoon climate, ferrallit soil (FAO classification; FAO, 2006) [5] and high humidity. The main soil properties were high content of iron and aluminum, hence the soil is acidity, thin layer and rocky.

FOREST MAPPING OF ME LINH STATION FOR BIODIVERSITY

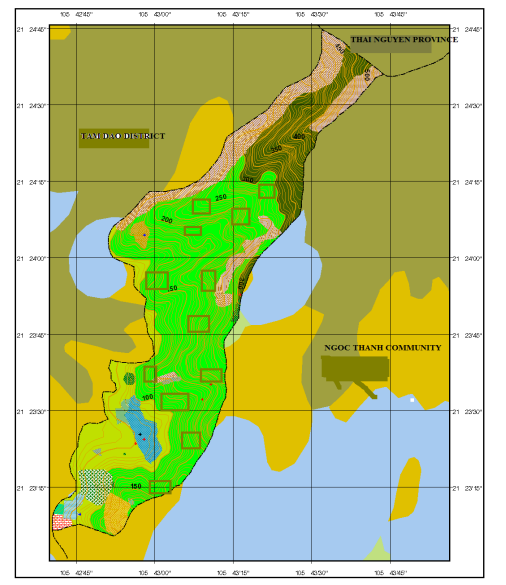

0.7 0.7 Kilometers

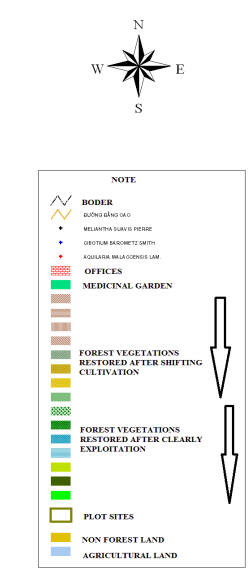

SCALE: $1 / 10,000$

Figure 1. Plots plan in Me Linh Biodiversity Station, Vinh Phuc province

Forest and Flora: the forests in the study area derived from tropical monsoon evergreen closed forest and they were significantly disturbed through exploiting activity of human, such as selective logging, clear cutting, grazing and collection of fuel-wood. Hence, most of the primary forests here are changed into two main secondary forest types: the secondary forest restored after shifting cultivation and the secondary forest restored after clearing cutting exploitation (Le Dong Tan, 2003) [8]. The area of the secondary forests restored after clear cutting exploitation is larger than that of the other type, about 85.7 ha $(48.01 \%$ of total area of the Station) and it is often distributed on the altitude of above $95 \mathrm{~m}$. The area of the secondary forest restored after shifting cultivation accounted about 38.9 ha $(21.79 \%)$ and distributed on the altitude of from $55 \mathrm{~m}$ to $79 \mathrm{~m}$.

The flora contains 166 families, 651 genera and 1129 species belonging to four divisions of higher vascular plant, including: Lycopodiophyta, Equisetophyta, Polypodiophyta, Magnoliophyta. The most abundant families are: Lauraceae, Fagaceae, Moraceae, Magnoliaceae, Fabaceae, Anacardiaceae, Burseraceae, Sapindaceae, Hamamelidaceae and Bombacaeae (Nguyen Tien Ban, 2000) [1]. 


\section{Plot selection}

Plots are selected in homogenous vegetation patches. Each plot has the size of $50 \mathrm{~m} \times 50 \mathrm{~m}$ (0.25ha plot), A total of ten plots is chosen to survey and to measure parameters in two forest types. In each of plot five subplots with the size of $(5 \mathrm{~m} \times 5 \mathrm{~m})$ have been created to survey and to collect data on shrub, grass and litter. The tasks performed in plots include collecting and identifying species specimen and measuring all trees with a diameter above 5 $\mathrm{cm}$ (at $1.3 \mathrm{~m}$ ) and higher $5 \mathrm{~m}$ in height.

\subsection{Methods of sampling}

\section{Selecting sample tree (represented tree)}

Tree is selected that is dominant species in each community with its diameter value in the medium of each diameter class (diameter parameter is divided into five classes such as class I: $5-10 \mathrm{~cm}$; class II: $>10-15 \mathrm{~cm}$; class $3:>15-$ $20 \mathrm{~cm}$; class 4: $>20-25 \mathrm{~cm}$ and class $5:>25 \mathrm{~cm})$. For one diameter class of each tree species we selected one sample tree to cut down and to calculate fresh biomass in the field as well as to take represented sample estimation dry biomass. So, the total number of samples accounted 100 individuals.

A tree is cut down close to the ground and divided into three parts: stem, branch and leave then is weighted to estimate the above ground fresh biomass. Root is a part of the tree to determine below ground fresh biomass. All the roots of a diameter of greater than $2 \mathrm{~mm}$ are dug and collected.

\section{Selecting sample in shrub and grass layer}

Samples of shrub and herbaceous plants are collected in each subplot. All shrubs and herbaceous plants are cut down and weighted to determine fresh biomass.

\section{Selecting sample for estimating ovendry biomass}

Dry biomass samples are taken from stem, branch, leave and root.

+ Stem: taking 3 different positions as top of stem, bottom of stem and bottom of the fork, cutting each position with a thickness of $3 \mathrm{~cm}$, the sample volume accounted for approximately $0.2 \%$ of the fresh weight.

+ Branch: taking 4 small cutting boards with total weight of $0.5 \mathrm{~kg}$.

+ Leave: mixing leaves and taking 01 sample of $0.5 \mathrm{~kg}$,
+ Root: taking a sample in tap-root and another in lateral root with total volume of $0.5 \mathrm{~kg}$.

All samples are cut small and dried in the oven at $70-85^{\circ} \mathrm{C}$ and the heating time depends on the part of plant body, but until gets constant weight.

\subsection{Data analysis}

Estimation of oven-dry biomass of tree/shrub/grass/litter by:

$$
M k i=M i \times \frac{m k_{i}}{m_{i}}
$$

Where:

Mki: oven-dry biomass of each part of individual; Mi: fresh biomass of each part of individual; mki: mass of dry sample of each part after drying and mi: mass of fresh sample of each part of individual

Estimation of dry biomass of represented tree (sampled tree) by:

$\mathrm{P}_{\mathrm{i}^{-} \text {tree }}=\mathrm{P}_{\mathrm{i}^{-} \text {stem }}+\mathrm{P}_{\mathrm{i}^{-} \text {branch }}+\mathrm{P}_{\mathrm{i}^{-} \text {leaf }}+\mathrm{P}_{\mathrm{i}^{-} \text {root }} \quad(\mathrm{kg})$

Where: $\mathrm{P}_{\mathrm{i}}$ : dry biomass of each part of sampled tree for a diameter class

\section{Biomass equations for tree}

A comprehensive review has been conducted for the biomass equations of 65 North American tree species (Michael T. Ter-Mikaelian and Michael D. Korzukhin (1997). All equations are of the form $\mathrm{M}=\mathrm{aDb}$, where $\mathrm{M}$ is the oven-dry weight of the biomass component of a tree $(\mathrm{kg})$, $\mathrm{D}$ is diameter at breast height $(\mathrm{DBH})(\mathrm{cm})$, and $\mathrm{a}$ and $\mathrm{b}$ are parameters. The equations for biomass estimation were developed by relating biomass to tree diameter $\left(\mathrm{D}_{1.3 \mathrm{~m}}\right)$ and other tree characteristics.

\section{Results and discussion}

\subsection{Biomass of sample tree}

Sample tree is chosen from dominant species in forests. Representatives of twenty dominant tree species are collected and cut down to account biomass of a tree of each species in each diameter class. Summarizing data on this biomass allowed to estimate average of each species and the results are shown in table 1. 
Table 1. Biomass of sample tree species in two forests in Me Linh Biodiversity Station

Scientific name $\begin{array}{lllll}\text { AGB } & \text { BGB } & \boldsymbol{\Sigma} \mathbf{P} & \mathbf{D}_{1.3 \mathrm{~m}} & \mathbf{H}\end{array}$

\begin{tabular}{llllll}
\hline 1. Hydnocarpus kurzii & 65.53 & 20.55 & 86.08 & 23.27 & 10.14
\end{tabular} (King) Warb.

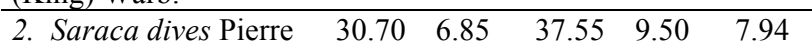

\begin{tabular}{llllll}
\hline 3. Machilus parviflora & 40.60 & 7.28 & 47.88 & 16.6 & 7.71
\end{tabular}

Meisn.

\begin{tabular}{llllll}
\hline 4. Diospyros eriantha & 55.65 & 13.68 & 69.34 & 13.5 & 9.03
\end{tabular}

Champ. ex Benth

$\begin{array}{llllll}\text { 5. Syzygium cinereum } & 68.55 & 18.61 & 87.16 & 10.90 & 7.92\end{array}$

Wall. ex Merr.

\begin{tabular}{llllll}
\hline 6. Aglaia spectabilis & 68.19 & 21.48 & 89.68 & 15.03 & 8.27
\end{tabular}

(Miq.) Jain

\begin{tabular}{lccccc}
\hline $\begin{array}{l}\text { 7. Engelhardtia rox- } \\
\text { burghiana Wall. }\end{array}$ & 37.57 & 14.48 & 52.05 & 19.17 & 7.66 \\
\hline $\begin{array}{l}\text { 8. Bischofia javanica } \\
\text { Blume }\end{array}$ & 61.95 & 13.20 & 75.15 & 20.6 & 14.29 \\
\hline $\begin{array}{l}\text { 9. Knema globularia } \\
\text { (Lamk.) Warrb. }\end{array}$ & 55.82 & 10.54 & 66.36 & 12.40 & 7.54 \\
\hline $\begin{array}{l}\text { lo. Litsea cubeba } \\
\text { (Lour.) Pers. }\end{array}$ & 92.02 & 19.43 & 111.4 & 23.73 & 11.95 \\
\end{tabular}

\begin{tabular}{|c|c|c|c|c|c|}
\hline Scientific name & AGB & BGB & $\Sigma P$ & $\mathbf{D}_{1.3 \mathrm{~m}}$ & $\mathbf{H}$ \\
\hline $\begin{array}{l}\text { 11. Wendlandia panicu- } \\
\text { lata (Roxb.) DC. }\end{array}$ & 23.22 & 6.90 & 30.12 & 10.77 & 6.93 \\
\hline $\begin{array}{l}\text { 12. Styrax tonkinensis } \\
\text { (Pierre) Craib. ex Hart- } \\
\text { wiss }\end{array}$ & 40.56 & 13.81 & 54.37 & 9.37 & 8.90 \\
\hline $\begin{array}{l}\text { 13. Liquidambar for- } \\
\text { mosana Hance }\end{array}$ & 52.62 & 12.78 & 65.40 & 11.64 & 7.35 \\
\hline $\begin{array}{l}\text { 14. Garcinia oblongifo- } \\
\text { lia Champ. ex Benth. }\end{array}$ & 67.13 & 13.31 & 80.44 & 16.80 & 8.82 \\
\hline $\begin{array}{l}\text { 15. Trema orientalis } \\
\text { (L.) Blume }\end{array}$ & 38.16 & 6.42 & 44.58 & 14.56 & 7.90 \\
\hline $\begin{array}{l}\text { 16. Duabanga grandi- } \\
\text { flora (Roxb. ex DC.) } \\
\text { Walp. }\end{array}$ & 59.17 & 10.82 & 69.99 & 15.05 & 8.92 \\
\hline $\begin{array}{l}\text { 17. Castanopsis indica } \\
\text { (Roxb.) A. DC. }\end{array}$ & 64.61 & 33.52 & 98.13 & 10.88 & 6.00 \\
\hline $\begin{array}{l}\text { 18. Aquilaria crassna } \\
\text { Pierre ex Lecomte }\end{array}$ & 92.58 & 32.67 & 125.2 & 9.02 & 6.70 \\
\hline $\begin{array}{l}\text { 19. Canarium } \\
\text { tonkinense Engl. }\end{array}$ & 41.86 & 8.97 & 50.83 & 9.95 & 7.77 \\
\hline $\begin{array}{l}\text { 20. Macaranga denticu- } \\
\text { lata (Blume) Muell.- } \\
\text { Arg. }\end{array}$ & 26.11 & 7.09 & 33.20 & 10.50 & 6.70 \\
\hline
\end{tabular}

Note: AGB: above ground biomass and BGB: Below ground biomass; Unit: kg.sample ${ }^{-1}$

The data in table 1 indicated that each tree species has its own average biomass like the biomass of Bischofia javanica achieved $75.15 \mathrm{~kg}$. tree ${ }^{-1}$; Macaranga denticulata (Blume) Muell.-Arg.: $33.20 \mathrm{~kg}$. tree ${ }^{-1}$; Liquidambar formosana Hance: $65.40 \mathrm{~kg}$. tree ${ }^{-1}$; etc.

In any of these tree species, the biomass identified mainly concentrated in AGB, approximately 60-75 percent of total biomass. For example: biomass of Engelhardtia roxburghiana Wall. divided into AGB: $37.57 \mathrm{~kg}^{- \text {tree }^{-1}}$ (72.18\%) and BGB: $14.48 \mathrm{~kg} \cdot \operatorname{tree}^{-1}(27.82 \%)$; biomass of Castanopsis indica (Roxb.) A. DC. Accounted AGB: $64.61 \mathrm{~kg} \cdot \operatorname{tree}^{-1}(65.84 \%)$ and BGB: 33.52 kg.tree ${ }^{-1}$ $(34.16 \%)$.

\subsection{Allometric relationships between bio- mass and two forest factors $\left(D_{1.3 m}\right.$ and $\left.H\right)$}

Experiment results on statistical software SPSS 16.0 showed that the relationship between biomass of the sample tree with $\mathrm{H}$ was simulated well with the main function equation $\mathrm{Y}=\mathrm{B}_{\mathrm{o}}+\mathrm{B}_{1} \mathrm{D}_{1.3}$ ( $\mathrm{Y}$ is the biomass).

3.2.1. Using Power function to identify allometric relation between biomass and diameter at the breast height of tree $\left(D_{1.3 m}\right)$.

A lot of research reports showed that individual-tree biomass estimates are usually based on a known allometric relationship between an easily measurable dimension (e.g., diameter at breast height) and biomass.

Table 2. Allometric equations between Fresh and Dry Biomass of tree species and diameter at the breast height $\left(D_{1.3 m}\right)$ in Me Linh Station for Biodiversity

\begin{tabular}{llllll} 
Scientific name & Allometric model & R & S & Sig.F & PT \\
\hline 1.Hydnocarpus kurzii (King) & $\mathrm{P}_{\text {fresh }}=22.42+1.12 \mathrm{D}_{1.3 \mathrm{~m}}$ & 0.957 & 0.169 & 0.000 & 4.1 \\
Warb. & $\mathrm{P}_{\text {dry }}=13.721+1.12 \mathrm{D}_{1.3 \mathrm{~m}}$ & 0.982 & 0.171 & 0.000 & 4.2 \\
& $\mathrm{P}_{\text {fresh }}=18.726+1.13 \mathrm{D}_{1.3 \mathrm{~m}}$ & 0.979 & 0.143 & 0.000 & 4.3 \\
2.Saraca dives Pierre & $\mathrm{P}_{\text {dry }}=9.5+1.13 \mathrm{D}_{1.3 \mathrm{~m}}$ & 0.968 & 0.144 & 0.000 & 4.4 \\
3.Machilus parviflora Meisn. & $\mathrm{P}_{\text {fresh }}=39.91+1.09 \mathrm{D}_{1.3 \mathrm{~m}}$ & 0.943 & 0.122 & 0.000 & 4.5 \\
& $\mathrm{P}_{\text {dry }}=22.48+1.09 \mathrm{D}_{1.3 \mathrm{~m}}$ & 0.902 & 0.129 & 0.000 & 4.6 \\
4.Diospyros eriantha Champ. & $\mathrm{P}_{\text {fresh }}=41.43+1 . \mathrm{D}_{1.3 \mathrm{~m}}$ & 0.936 & 0.159 & 0.000 & 4.7 \\
ex Benth & $\mathrm{P}_{\text {dry }}=23.65+1.09 \mathrm{D}_{1.3 \mathrm{~m}}$ & 0.969 & 0.156 & 0.000 & 4.8 \\
5.Syzygium cinereum Wall. ex & $\mathrm{P}_{\text {fresh }}=36.03+1 . \mathrm{D}_{1.3 \mathrm{~m}}$ & 0.981 & 0.106 & 0.000 & 4.9 \\
Merr. & $\mathrm{P}_{\text {dry }}=15.4+1.1 \mathrm{D}_{1.3 \mathrm{~m}}$ & 0.980 & 0.114 & 0.000 & 4.10
\end{tabular}




\begin{tabular}{|c|c|c|c|c|c|}
\hline Scientific name & Allometric model & $\mathbf{R}$ & $\mathbf{S}$ & Sig.F & PT \\
\hline \multirow[t]{2}{*}{ 6.Aglaia spectabilis (Miq.) Jain } & $\mathrm{P}_{\text {fresh }}=55.81+1.085 \mathrm{D}_{1.3 \mathrm{~m}}$ & 0.986 & 0.111 & 0.000 & 4.11 \\
\hline & $P_{\text {dry }}=26.74+1.092 \mathrm{D}_{1.3 \mathrm{~m}}$ & 0.969 & 0.121 & 0.000 & 4.12 \\
\hline \multirow{2}{*}{$\begin{array}{l}\text { 7.Engelhardtia roxburghiana } \\
\text { Wall. }\end{array}$} & $\mathrm{P}_{\text {fresh }}=24.1+1.121 \mathrm{D}_{1.3 \mathrm{~m}}$ & 0.987 & 0.111 & 0.000 & 4.13 \\
\hline & $\mathrm{P}_{\mathrm{dry}}=11.529+1.127 \mathrm{D}_{1.3 \mathrm{~m}}$ & 0.981 & 0.118 & 0.000 & 4.14 \\
\hline \multirow[t]{2}{*}{ 8.Bischofia javanica Blume } & $\mathrm{P}_{\text {fresh }}=23.74+1.12 \mathrm{D}_{1.3 \mathrm{~m}}$ & 0.988 & 0.124 & 0.000 & 4.15 \\
\hline & $\mathrm{P}_{\mathrm{dry}}=10.487+1.127 \mathrm{D}_{1.3 \mathrm{~m}}$ & 0.990 & 0.127 & 0.000 & 4.16 \\
\hline \multirow{2}{*}{$\begin{array}{l}\text { 9.Knema globularia (Lamk.) } \\
\text { Warrb. }\end{array}$} & $\mathrm{P}_{\text {fresh }}=35.99+1.113 \mathrm{D}_{1.3 \mathrm{~m}}$ & 0.985 & 0.109 & 0.000 & 4.17 \\
\hline & $\mathrm{P}_{\text {dry }}=20.26+1.116 \mathrm{D}_{1.3 \mathrm{~m}}$ & 0.976 & 0.109 & 0.000 & 4.18 \\
\hline \multirow{2}{*}{ 10.Litsea cubeba (Lour.) Pers. } & $\mathrm{P}_{\text {fresh }}=28.78+1.126 \mathrm{D}_{1.3 \mathrm{~m}}$ & 0.969 & 0.095 & 0.000 & 4.19 \\
\hline & $\mathrm{P}_{\text {dry }}=12.196+1.133 \mathrm{D}_{1.3 \mathrm{~m}}$ & 0.989 & 0.104 & 0.000 & 4.20 \\
\hline \multirow{2}{*}{$\begin{array}{l}\text { 11. Wendlandia paniculata } \\
\text { (Roxb.) DC. }\end{array}$} & $P_{\text {fresh }}=22.49+1.113 \mathrm{D}_{1.3 \mathrm{~m}}$ & 0.988 & 0.087 & 0.000 & 4.21 \\
\hline & $\mathrm{P}_{\mathrm{dry}}=12.69+1.116 \mathrm{D}_{1.3 \mathrm{~m}}$ & 0.985 & 0.091 & 0.000 & 4.22 \\
\hline \multirow{2}{*}{$\begin{array}{l}\text { 12.Styrax tonkinensis (Pierre) } \\
\text { Craib. ex Hartwiss }\end{array}$} & $\mathrm{P}_{\text {fresh }}=17.84+1.126 \mathrm{D}_{1.3 \mathrm{~m}}$ & 0.988 & 0.105 & 0.000 & 4.23 \\
\hline & $\mathrm{P}_{\text {dry }}=8.52+1.131 \mathrm{D}_{1.3 \mathrm{~m}}$ & 0.986 & 0.108 & 0.000 & 4.24 \\
\hline \multirow{2}{*}{$\begin{array}{l}\text { 13.Liquidambar formosana } \\
\text { Hance }\end{array}$} & $P_{\text {fresh }}=29.21+1.12 \mathrm{D}_{1.3 \mathrm{~m}}$ & 0.966 & 0.167 & 0.000 & 4.25 \\
\hline & $\mathrm{P}_{\mathrm{dry}}=18.884+1.123 \mathrm{D}_{1.3 \mathrm{~m}}$ & 0.967 & 0.169 & 0.000 & 4.26 \\
\hline \multirow{2}{*}{$\begin{array}{l}\text { 14.Garcinia oblongifolia } \\
\text { Champ. ex Benth. }\end{array}$} & $\mathrm{P}_{\text {fresh }}=41.38+1.111 \mathrm{D}_{1.3 \mathrm{~m}}$ & 0.980 & 0.128 & 0.000 & 4.27 \\
\hline & $\begin{array}{l}\mathrm{P}_{\text {dry }}=26.649+1.116 \mathrm{D}_{1.3 \mathrm{~m}} \\
\mathrm{P}_{\text {fresh }}=32.04+1.127 \mathrm{D}_{1.3 \mathrm{~m}}\end{array}$ & $\begin{array}{l}0.978 \\
0.973\end{array}$ & $\begin{array}{l}0.140 \\
0.151\end{array}$ & $\begin{array}{l}0.000 \\
0.000\end{array}$ & $\begin{array}{l}4.28 \\
4.29\end{array}$ \\
\hline \multirow[t]{2}{*}{ 15.Trema orientalis (L.) Blume } & $P_{\text {dry }}=17.38+1.13 \mathrm{D}_{1.3 \mathrm{~m}}$ & 0.951 & 0.219 & 0.000 & 4.30 \\
\hline & $\mathrm{P}_{\text {fresh }}=18.79+1.029 \mathrm{D}_{1.3 \mathrm{~m}}$ & 0.957 & 0.059 & 0.000 & 4.31 \\
\hline \multirow{3}{*}{$\begin{array}{l}\text { 16.Duabanga grandiflora } \\
\text { (Roxb. ex DC.) Walp. } \\
\text { 17.Castanopsis indica (Roxb.) } \\
\text { A. DC. ex Lecomte }\end{array}$} & $\mathrm{P}_{\mathrm{dry}}=10.175+1.103 \mathrm{D}_{1.3 \mathrm{~m}}$ & 0.963 & 0.107 & 0.000 & 4.32 \\
\hline & $\mathrm{P}_{\text {fresh }}=25.68+1.027 \mathrm{D}_{1.3 \mathrm{~m}}$ & 0.988 & 0.115 & 0.000 & 4.33 \\
\hline & $\mathrm{P}_{\mathrm{dry}}=19.279+1.055 \mathrm{D}_{1.3 \mathrm{~m}}$ & 0.968 & 0.167 & 0.000 & 4.34 \\
\hline \multirow[t]{2}{*}{ 18.Aquilaria crassna Pierre } & $\mathrm{P}_{\text {fresh }}=32.06+2.001 \mathrm{D}_{1.3 \mathrm{~m}}$ & 0.977 & 0.099 & 0.000 & 4.35 \\
\hline & $\mathrm{P}_{\mathrm{dry}}=24.096+1.503 \mathrm{D}_{1.3 \mathrm{~m}}$ & 0.980 & 0.113 & 0.000 & 4.36 \\
\hline \multirow[t]{2}{*}{ 19.Canarium tonkinense Engl. } & $\mathrm{P}_{\text {fresh }}=19.08+2.028 \mathrm{D}_{1.3 \mathrm{~m}}$ & 0.936 & 0.167 & 0.000 & 4.37 \\
\hline & $\mathrm{P}_{\mathrm{dry}}=11.015+1.227 \mathrm{D}_{1.3 \mathrm{~m}}$ & 0.950 & 0.145 & 0.000 & 4.38 \\
\hline \multirow{2}{*}{$\begin{array}{l}\text { 20.Macaranga denticulata } \\
\text { (Blume) Muell.-Arg. }\end{array}$} & $\mathrm{P}_{\mathrm{fresh}}=14.082+2.061 \mathrm{D}_{1.3 \mathrm{~m}}$ & 0.978 & 0.128 & 0.000 & 4.39 \\
\hline & $\mathrm{P}_{\mathrm{dry}}=12.330+1.817 \mathrm{D}_{1.3 \mathrm{~m}}$ & 0.980 & 0.166 & 0.000 & 4.50 \\
\hline
\end{tabular}

For one tree species two equations have been established to find out allometric between fresh biomass or dry biomass of tree and $D_{1.3 \mathrm{~m}}$. All of forty regression equations in table 2 show that correlation coefficients recorded the lowest value was $0.902\left(\mathrm{R}^{2}<0.902\right)$, such as: Fresh and dry Biomass of Aquilaria crassna were expressed strongly relation with $\mathrm{D}_{1.3 \mathrm{~m}}\left(\mathrm{R}^{2}: 0.977\right.$ and 0.980$)$; Styrax tonkinensis $\left(\mathrm{R}^{2}\right.$ : 0.988 and 0.986); Machilus parviflora $\left(\mathrm{R}^{2}: 0.943\right.$ and 0.902$)$. It is suggested that all the biomass of tree species in the natural vegetation at the Me Linh Biodiversity Station is strongly correlated with the diameter factor. When the stem diameter increases so biomass will also increase.

3.2.2. Using Power function to simulate allometric relation between biomass and height of tree $(\mathrm{H})$.
Tree heights can be difficult to measure under the best conditions. Height measurements are dependent on forest conditions, observer experience, and the equipment used. Tropical forests typically include significant obstacles for traditional field-based estimates of tree heights, including dense understory vegetation, tall canopies, and closedcanopy conditions that limit the line of sight. Tree height measurements in tropical forests both are labor intensive and have potentially large errors. Although researchers agree that height is a valuable addition when estimating biomass, the degree of acceptable error has been debated. We evaluate how tree height accuracy affects biomass estimation accuracy for moist tropical forests in Me Linh Station for Biodiversity by the allometric equations in table 3 . 
Table 3. Allometric equations between Fresh and Dry Biomass of tree species and height of tree (H) in Me Linh Station for Biodiversity

\begin{tabular}{|c|c|c|c|c|c|}
\hline Scientific name & Allometric model & $\mathbf{R}$ & $\mathbf{S}$ & Sig.F & PT \\
\hline \multirow{3}{*}{$\begin{array}{l}\text { 1.Hydnocarpus kurzii } \\
\text { Warb. }\end{array}$} & $\mathrm{P}_{\text {fresh }}=10.93+0.29 \mathrm{H}$ & 0.483 & 0.02 & 0.000 & 2.2 \\
\hline & $\mathrm{P}_{\mathrm{dry}}=8.001+0.82 \mathrm{H}$ & 0.501 & 0.171 & 0.000 & 2.7 \\
\hline & $\mathrm{P}_{\text {fresh }}=8.726+1.13 \mathrm{H}$ & 0.502 & 0.143 & 0.000 & 1.8 \\
\hline 2.Saraca dives Pierre & $\mathrm{P}_{\mathrm{dry}}=9.5+1.13 \mathrm{H}$ & 0.425 & 0.144 & 0.000 & 2.0 \\
\hline \multirow[t]{2}{*}{ 3.Machilus parviflora Meisn. } & $\mathrm{P}_{\text {fresh }}=9.82+1.68 \mathrm{H}$ & 0.328 & 0.122 & 0.000 & 2.0 \\
\hline & $\mathrm{P}_{\mathrm{dry}}=11.57+1.09 \mathrm{H}$ & 0.522 & 0.129 & 0.000 & 2.4 \\
\hline \multirow{2}{*}{$\begin{array}{l}\text { 4.Diospyros eriantha Champ. ex } \\
\text { Benth }\end{array}$} & $\mathrm{P}_{\text {fresh }}=11.33+1.0 \mathrm{H}$ & 0.364 & 0.120 & 0.000 & 1.7 \\
\hline & $\mathrm{P}_{\mathrm{dry}}=5.65+0.39 \mathrm{H}$ & 0.52 & 0.106 & 0.000 & 2.8 \\
\hline \multirow{2}{*}{$\begin{array}{l}\text { 5.Syzygium cinereum Wall. ex } \\
\text { Merr. }\end{array}$} & $P_{\text {fresh }}=146.23+1.0 \mathrm{H}$ & 0.503 & 0.106 & 0.000 & 2.2 \\
\hline & $\mathrm{P}_{\text {dry }}=5.7+0.908 \mathrm{H}$ & 0.482 & 0.135 & 0.000 & 2.10 \\
\hline \multirow[t]{2}{*}{ 6.Aglaia spectabilis (Miq.) Jain } & $\mathrm{P}_{\text {fresh }}=15.01+0.85 \mathrm{H}$ & 0.460 & 0.122 & 0.000 & 2.1 \\
\hline & $\mathrm{P}_{\mathrm{dry}}=16.40+0.95 \mathrm{H}$ & 0.471 & 0.101 & 0.000 & 2.12 \\
\hline \multirow[t]{2}{*}{ 7.Engelhardtia roxburghiana Wall. } & $\mathrm{P}_{\text {fresh }}=14.3+0.90 \mathrm{H}$ & 0.487 & 0.105 & 0.000 & 2.13 \\
\hline & $\mathrm{P}_{\text {dry }}=10.9+1.07 \mathrm{H}$ & 0.455 & 0.119 & 0.000 & 2.14 \\
\hline \multirow[t]{2}{*}{ 8.Bischofia javanica Blume } & $\mathrm{P}_{\text {fresh }}=10.64+1.02 \mathrm{H}$ & 0.480 & 0.117 & 0.000 & 2.4 \\
\hline & $\mathrm{P}_{\mathrm{dry}}=5.55+1.7 \mathrm{H}$ & 0.492 & 0.127 & 0.000 & 2.3 \\
\hline \multirow{2}{*}{$\begin{array}{l}\text { 9.Knema globularia (Lamk.) } \\
\text { Warrb. }\end{array}$} & $\mathrm{P}_{\text {fresh }}=15.0+0.93 \mathrm{H}$ & 0.486 & 0.109 & 0.000 & 2.0 \\
\hline & $\mathrm{P}_{\mathrm{dry}}=10.06+0.6 \mathrm{H}$ & 0.476 & 0.189 & 0.000 & 2.4 \\
\hline \multirow[b]{2}{*}{ 10.Litsea cubeba (Lour.) Pers. } & $P_{\text {fresh }}=719+0.65 \mathrm{H}$ & 0.440 & 0.080 & 0.000 & 2.5 \\
\hline & $\mathrm{P}_{\mathrm{dry}}=8.96+1.63 \mathrm{H}$ & 0.409 & 0.104 & 0.000 & 2.2 \\
\hline \multirow{2}{*}{$\begin{array}{l}\text { 11.Wendlandia paniculata (Roxb.) } \\
\text { DC. }\end{array}$} & $\mathrm{P}_{\text {fresh }}=12.4+1.3 \mathrm{H}$ & 0.440 & 0.087 & 0.000 & 2.3 \\
\hline & $\mathrm{P}_{\text {dry }}=12.15+0.61 \mathrm{H}$ & 0.471 & 0.082 & 0.000 & 1.9 \\
\hline \multirow{2}{*}{$\begin{array}{l}\text { 12.Styrax tonkinensis } \\
\text { Craib. ex Hartwiss }\end{array}$} & $\mathrm{P}_{\text {fresh }}=10.22+.92 \mathrm{H}$ & 0.380 & 0.129 & 0.000 & 2.2 \\
\hline & $\mathrm{P}_{\mathrm{dry}}=8.052+1.26 \mathrm{H}$ & 0.503 & 0.145 & 0.000 & 2.4 \\
\hline \multirow[t]{2}{*}{ 13.Liquidambar formosana Hance } & $\mathrm{P}_{\text {fresh }}=14.0+1.12 \mathrm{H}$ & 0.499 & 0.133 & 0.000 & 2.3 \\
\hline & $\mathrm{P}_{\mathrm{dry}}=7.55+0.84 \mathrm{H}$ & 0.552 & 0.145 & 0.000 & 2.2 \\
\hline \multirow{3}{*}{$\begin{array}{l}\text { 14.Garcinia oblongifolia Champ. } \\
\text { ex Benth. }\end{array}$} & $\mathrm{P}_{\text {fresh }}=4.34+0.8 \mathrm{H}$ & 0.487 & 0.130 & 0.000 & 2.0 \\
\hline & $\mathrm{P}_{\mathrm{dry}}=6.05+0.66 \mathrm{H}$ & 0.433 & 0.148 & 0.000 & 1.7 \\
\hline & $\mathrm{P}_{\text {fresh }}=12.04+0.72 \mathrm{H}$ & 0.502 & 0.151 & 0.000 & 1.9 \\
\hline \multirow[t]{2}{*}{ 15.Trema orientalis (L.) Blume } & $\mathrm{P}_{\text {dry }}=7.38+0.32 \mathrm{H}$ & 0.511 & 0.212 & 0.000 & 2.5 \\
\hline & $\mathrm{P}_{\text {fresh }}=7.92+0.29 \mathrm{H}$ & 0.533 & 0.101 & 0.000 & 1.9 \\
\hline \multirow{3}{*}{$\begin{array}{l}\text { 16.Duabanga grandiflora (Roxb. } \\
\text { ex DC.) Walp. } \\
\text { 17.Castanopsis indica (Roxb.) A. } \\
\text { DC.ex Lecomte }\end{array}$} & $\mathrm{P}_{\mathrm{dry}}=10.175+0.88 \mathrm{H}$ & 0.505 & 0.107 & 0.000 & 2.4 \\
\hline & $\mathrm{P}_{\text {fresh }}=15.55+07 \mathrm{H}$ & 0.476 & 0.109 & 0.000 & 2.3 \\
\hline & $\mathrm{P}_{\mathrm{dry}}=19.279+1.055 \mathrm{H}$ & 0.557 & 0.166 & 0.000 & 2.2 \\
\hline \multirow[t]{2}{*}{ 18.Aquilaria crassna Pierre } & $\mathrm{P}_{\text {fresh }}=9.06+1.12 \mathrm{H}$ & 0.300 & 0.076 & 0.000 & 1.9 \\
\hline & $\mathrm{P}_{\mathrm{dry}}=14.61+0.6 \mathrm{H}$ & 0.302 & 0.143 & 0.000 & 2.3 \\
\hline \multirow[t]{2}{*}{ 19.Canarium tonkinense Engl. } & $\mathrm{P}_{\text {fresh }}=10.01+0.81 \mathrm{H}$ & 0.543 & 0.151 & 0.000 & 2.5 \\
\hline & $\mathrm{P}_{\text {dry }}=10.15+0.87 \mathrm{H}$ & 0.409 & 0.143 & 0.000 & 2.3 \\
\hline \multirow{2}{*}{$\begin{array}{l}\text { 20.Macaranga denticulata (Blume) } \\
\text { Muell.-Arg. }\end{array}$} & $\mathrm{P}_{\text {fresh }}=9.00+0.61 \mathrm{H}$ & 0.488 & 0.104 & 0.000 & 2.0 \\
\hline & $\mathrm{P}_{\text {dry }}=9.0+0.55 \mathrm{H}$ & 0.455 & 0.166 & 0.000 & 2.2 \\
\hline
\end{tabular}

The table 3 showed that the correlation between fresh and dry biomass with the tree height of 20 species of sample trees. The correlation coefficient of 40 regression equations was relatively low, the highest $\mathrm{R}$ value was 0.549 of 
Garcinia oblongifolia Champ. ex Benth and almost these values were vary from 0.403 to 0.502 , including some species: Liquidambar formosana Hance, Bischofia javanica Blume, Canarium tonkinense Engl, ect. This is true of the status of natural forests cover in the research site, in which almost young forest areas have been recovered from shifting cultivation and have been exploited for the past 20 years. Therefore, the tree height is still low, ranging from $5.9 \mathrm{~m}$ to $10.3 \mathrm{~m}$ and the trees are unstable both in height and the species composition.

\subsubsection{Using Power Function to find out allometric relation between tree biomass and two factors $\left(D_{1.3 \mathrm{~m}}\right.$, H)}

Based on the above results, correlations of biomass of tree parts (such as: stem, branch, leaves and root) and two forest factors $\left(\mathrm{D}_{1.3 \mathrm{~m}}\right.$ and $\left.\mathrm{H}\right)$ were tested and the results are shown in table 4.

Table 4. The correlation between above ground biomass $\left(P_{\mathrm{AGB}}\right)$ of tree parts (stem, branch, leaves) and two forest factors

\begin{tabular}{ccrrrrrr} 
Indicator & Statistic & $\mathbf{D}_{\mathbf{1 . 3 m}} \mathbf{( c m )}$ & $\mathbf{H}(\mathbf{m})$ & $\begin{array}{r}\mathbf{P}_{\text {AGB. stem }} \\
\mathbf{( k g})\end{array}$ & $\begin{array}{r}\mathbf{P}_{\text {AGB. }} \\
\text { branch }\end{array} \mathbf{( k g )}$ & $\begin{array}{r}\mathbf{P}_{\text {AGB. leaves }} \\
(\mathbf{k g})\end{array}$ & $\begin{array}{r}\text { Total bio- } \\
\text { mass (kg) }\end{array}$ \\
\hline \multirow{2}{*}{$\mathrm{D}_{1.3 \mathrm{~m}}(\mathrm{~cm})$} & $\mathrm{R} 2$ & - & 0.9743 & 0.9817 & 0.8914 & 0.9061 & 0.9338 \\
& $\mathrm{P}$ & - & 0.001 & 0.001 & 0.001 & 0.001 & 0.001 \\
$\mathrm{H}(\mathrm{m})$ & $\mathrm{R} 2$ & 0.9743 & - & 0.6311 & 0.4826 & 0.5023 & 0.4919 \\
& $\mathrm{P}$ & 0.001 & - & 0.001 & 0.001 & 0.001 & 0.001
\end{tabular}

The correlation coefficient of above ground biomass of parts was recorded closed corelation with diameter at the breast height $\left(\mathrm{D}_{1.3 \mathrm{~m}}\right)\left(\mathrm{R}_{\text {total biomass }}^{2}: 0.9338 ; \mathrm{R}^{2} \mathrm{P}_{\mathrm{AGB} \text {. stem }}\right.$ :
0.9817; $\mathrm{R}^{2} \mathrm{P}_{\mathrm{AGB}}$ branch $0.8914 ; \mathrm{R}^{2} \mathrm{P}_{\mathrm{AGB}}$, leaves: 0.9061; $\mathrm{P}<0.001$ ) but the relation between biomass and tree height is not clear $\left(\mathrm{R}^{2}<0.4919\right)$ as shown in table 5 .

Table 5. Biomass Models for tree species in Me Linh Biodiversity Station

\begin{tabular}{|c|c|c|c|c|c|}
\hline No. & Scientific name & Biomass equations & No. & Scientific name & Biomass equations \\
\hline \multirow[t]{2}{*}{1} & Hydnocarpus kurzii & $\mathrm{P}=11.6 . \mathrm{D}^{2.781} \mathrm{H}$ & 11 & Wendlandia & $\mathrm{P}=9.29 \mathrm{D}^{2.660} \mathrm{H}$ \\
\hline & (King) Warb. & $\mathrm{R}^{2}: 0.5583$ & & (Roxb.) DC. & $\mathrm{R}^{2}: 0.4066$ \\
\hline \multirow[t]{2}{*}{2} & & $\mathrm{P}=19.91 \mathrm{D}^{1.104} \mathrm{H}$ & 12 & Styrax tonkinensis (Pierre) & $\mathrm{P}=13.59 \mathrm{D}^{1.589} \mathrm{H}$ \\
\hline & Saraca dives Pierre & $\mathrm{R}^{2}: 0.802$ & & Craib. ex Hartwiss & $\mathrm{R}^{2}: 0.4557$ \\
\hline \multirow[t]{2}{*}{3} & parviflora & $\mathrm{P}=10.05 \mathrm{D}^{2.668} \mathrm{H}$ & 13 & \multirow{2}{*}{$\begin{array}{l}\text { Liquidambar } \\
\text { Hance }\end{array}$} & $\mathrm{P}=11.95 \mathrm{D}^{2.058}$ \\
\hline & Meisn. & $\mathrm{R}^{2}: 0.3052$ & & & $\mathrm{R}^{2}: 0.5022$ \\
\hline \multirow[t]{2}{*}{4} & Dic & $\mathrm{P}=15.56 \mathrm{D}^{0.998} \mathrm{H}$ & 14 & Garcinia oblongifolia & $\mathrm{P}=8.771 \mathrm{D}^{1.614} \mathrm{H}$ \\
\hline & Champ. ex Benth & $\mathrm{R}^{2}: 0.5809$ & & Champ. ex Benth. & $\mathrm{R}^{2}: 0.6111$ \\
\hline \multirow[t]{2}{*}{5} & Syzygium & $\mathrm{P}=9.794 \mathrm{D}^{2.450} \mathrm{H}$ & 15 & \multirow{2}{*}{ Trema orientalis (L.) } & $\mathrm{P}=1.550 \mathrm{D}^{2.290} \mathrm{H}$ \\
\hline & Wall. ex Merr. & $\mathrm{R}^{2}: 0.6770$ & & & $\mathrm{R}^{2}: 0.4400$ \\
\hline \multirow[t]{2}{*}{6} & spectabilis & $\mathrm{P}=3.629 \mathrm{D}^{1.776} \mathrm{H}$ & 16 & \multirow{2}{*}{$\begin{array}{l}\text { Duabanga grandiflora } \\
\text { (Roxb. ex DC.) Walp. }\end{array}$} & $\mathrm{P}=6.403 \mathrm{D}^{2.037} \mathrm{H}$ \\
\hline & (Miq.) Jain & $\mathrm{R}^{2}: 0.4077$ & & & $\mathrm{R}^{2}: 0.5089$ \\
\hline \multirow[t]{2}{*}{7} & Engelh & $\mathrm{P}=22.845 \mathrm{D}^{1.007} \mathrm{H}$ & 17 & \multirow{2}{*}{$\begin{array}{l}\text { Castanopsis } \\
\text { (Roxb.) A. DC. }\end{array}$} & $\mathrm{P}=7.05 \mathrm{D}^{2.668} \mathrm{H}$ \\
\hline & burghiana Wall. & $\mathrm{R}^{2}: 0.9020$ & & & $\mathrm{R}^{2}: 0.6540$ \\
\hline \multirow[t]{2}{*}{8} & Bischofia & $\mathrm{P}=21.239 \mathrm{D}^{2.001} \mathrm{H}$ & 18 & \multirow[t]{2}{*}{ Aquilaria crassna Pierre } & $\mathrm{P}=12.103 \mathrm{D}^{1.66} \mathrm{H}$ \\
\hline & Blume & $\mathrm{R}^{2}: 0.3881$ & & & $\mathrm{R}^{2}: 0.3380$ \\
\hline \multirow[t]{2}{*}{9} & globularia & $\mathrm{P}=6.905 \mathrm{D}^{1.115} \mathrm{H}$ & 19 & tonkinense & $\mathrm{P}=1.998 . \mathrm{D}^{0.557} \mathrm{H}$ \\
\hline & (Lamk.) Warrb. & $\mathrm{R}^{2}: 0.6333$ & & Engl. & $\mathrm{R}^{2}: 0.7031$ \\
\hline \multirow[t]{2}{*}{10} & Litsea cubeba & $\mathrm{P}=15.790 \mathrm{D}^{2.643} \mathrm{H}$ & 20 & Macaranga & $\mathrm{P}=3.008 . \mathrm{D}^{0.99} \mathrm{H}$ \\
\hline & Pers. & $\mathrm{R}^{2}: 0.8112$ & & (Blume) Muell.-Arg. & $\mathrm{R}^{2}: 0.5720$ \\
\hline
\end{tabular}

Table 5 presents twenty allometric models that were designed for estimating tree biomass of 20 species based on two forest factors (D and H). However, some species showed close correlation such as: Saraca dives Pierre, Engelhardtia roxburghiana Wall., Litsea cubeba (Lour.)
Pers. Canarium tonkinense Engl. $\left(\mathrm{R}^{2}>0.7031\right)$. For other species it was not clearly or weak correlation, including: Bischofia javanica Blume, Machilus parviflora Meisn., Styrax tonkinensis (Pierre) Craib. ex Hartwiss, ect. 


\section{Conclusion}

As the results show the allometric equations gives the most correlation coefficient between biomass and diameter inventory factors higher than $0.8589\left(\mathrm{R}^{2}>0.8589\right.$. $\mathrm{P}<$ 0.0001 ) and expressed that this is closed relation. However, the allometric between biomass and the height factor is still showed clearly and continue test.

Four allometric equations were established to estimate natural forest biomass in Me Linh Station for Biodiversity:

$\mathrm{P}_{\text {stem }}=25.3051 *\left(\mathrm{D}_{1.3 \mathrm{~m}}\right)^{0.4627}\left(\mathrm{R}^{2}: 9.661\right)$

$\mathrm{P}_{\text {branch }}=12.1043 *\left(\mathrm{D}_{1.3 \mathrm{~m}}\right)^{0.5416}\left(\mathrm{R}^{2}: 9.8\right)$

$\mathrm{P}_{\text {leaves }}=9.446 *\left(\mathrm{D}_{1.3 \mathrm{~m}}\right)^{0.5976}\left(\mathrm{R}^{2}: 0.9363\right)$

$\mathrm{P}_{\text {toatla biomass forest }}=25.882 * \mathrm{D}^{1.725}$ with $\left.\mathrm{R}^{2}: 0.8561\right)$

All equations will be applied to estimate biomass for each tree species or natural forest in Me Linh Station for Biodiversity in recently.

Acknowledgement: this study has been done with the financial support from a grant of the project: "Research on biomass carbon stock of tree species in natural forest in Me Linh Station for Biodiversity. Vinh Phuc province”. coded IEBR ĐT 13/18.

\section{References}

[1] Nguyen Tien Ban, 2000. Flora in Me Linh Biodiversity Station.

[2] Bao Huy, Pham Tuan Anh, Vo Hung and Nguyen Thi Thanh Huong (2012). Report on estimation of $\mathrm{CO} 2$ sequestered in evergreen broad leaf forest in Central Highland of Vietnam for reducing deforestation and forest degradation program. Tay Nguyen University, Dak Lac.
[3] Brown. S. and A. E. Lugo. 1992. Above ground biomass estimates for tropical moist forests of the Brazilian Amazon. Interciencia 17: 8-18.

[4] Chave. J. Andalo. C. Brown. S. Cairns. M.A. Chambers. J.Q. Eamus. D. Folster. H. Fromard. F. Higuchi. N. Kira. T. Lescure. J.P. Nelson. B.W. Ogawa. H. Puig. H. Rie'ra. B. And Yamakura. T. (2005). Tree allometry and improved estimation of carbon stocks and balance in tropical forests. Oecologia 145: 87-99.

[5] FAO. 2004. A review of carbon sequestration projects. Rome. 2004. Pines: drawings and descriptions of the genus Pinus. Leiden: Brill \& Backhuys.

[6] International Classification and Mapping of Vegetation. 1973. Published by the United Nations Educational. Scientific and Cultural Organization. UNESCO. Paris. 93p.

[7] IPCC. 2006. Guideline for National Greenhouse Gas Inventories. Volume 4: Agriculture. Forestry and Other Land Use. Intergovernmental Panel on Climate Change (IPCC). IPCC/OECD/IEA. Paris. France.

[8] Le Dong Tan, 2003. The status of forests in Me Linh Biodiversity Station.

[9] Raae, K., Christensen, P.C., Vu Tan Phuong and Vu Tien Dien (2010). Technical Report on Technical Assistance in the Development of the National REDD Programme of Vietnam: Component of Collecting Information and Analyzing Trends of Forest Resources and Forest Carbon.

[10] Timothy R.H. Pearson, Sandra L. Brown, Richard A. Birdsey. 2007. Measurement Guidelines for the sequestration forest carbon. Gen. Tech. Rep. NRS-18. Newtown Square, PA: U.S. Department of Agriculture, Forest Service, Northern Research Station. 42 p. 